\title{
Artificial Intelligence in Upper Gastrointestinal Endoscopy
}

\author{
Meltem Tokat Laurelle van Tilburg Arjun D. Koch Manon C.W. Spaander \\ Department of Gastroenterology and Hepatology, Erasmus MC Cancer Institute, University Medical Center \\ Rotterdam, Rotterdam, The Netherlands
}

\section{Keywords}

Artificial intelligence - Deep learning - Esophageal cancer ·

Gastric cancer · Endoscopy

\begin{abstract}
Background: Over the past decade, several artificial intelligence (Al) systems are developed to assist in endoscopic assessment of (pre-)cancerous lesions of the gastrointestinal (GI) tract. In this review, we aimed to provide an overview of the possible indications of $\mathrm{Al}$ technology in upper $\mathrm{Gl}$ endoscopy and hypothesize about potential challenges for its use in clinical practice. Summary: Application of Al in upper GI endoscopy has been investigated for several indications: (1) detection, characterization, and delineation of esophageal and gastric cancer (GC) and their premalignant conditions; (2) prediction of tumor invasion; and (3) detection of Helicobacter pylori. Al systems show promising results with an accuracy of up to $99 \%$ for the detection of superficial and advanced upper $\mathrm{Gl}$ cancers. Al outperformed trainee and experienced endoscopists for the detection of esophageal lesions and atrophic gastritis. For GC, Al outperformed mid-level and trainee endoscopists but not expert endoscopists. Key Messages: Application of artificial intelligence (Al) in upper gastrointestinal endoscopy may improve early diagnosis of esophageal and gastric cancer and may enable endoscopists
\end{abstract}

karger@karger.com www.karger.com/ddi

Karger"

GOPEN ACCESS
(C) 2021 The Author(s)

Published by S. Karger AG, Basel

This is an Open Access article licensed under the Creative Common Attribution-NonCommercial-4.0 International License (CC BY-NC) (http://www.karger.com/Services/OpenAccessLicense), applicable to the online version of the article only. Usage and distribution for commercial purposes requires written permission. to better identify patients eligible for endoscopic resection. The benefit of Al on the quality of upper endoscopy still needs to be demonstrated, while prospective trials are needed to confirm accuracy and feasibility during real-time daily endoscopy.

(c) 2021 The Author(s)

Published by S. Karger AG, Basel

\section{Introduction}

Accurate endoscopic detection of esophageal and gastric cancers and their premalignant conditions, such as Barrett neoplasia, gastric atrophy, and intestinal metaplasia, is essential for the detection of these cancers at an early stage [1-4]. The challenge of endoscopic procedures lies in the real-time interpretation of endoscopic imagery, which is complex and sensitive to human error. Current endoscopic cancer screening and surveillance strategies encounter several pitfalls, including interobserver variability in the detection of lesions, time-consuming biopsy protocols, and biopsy sampling error $[1,5,6]$. Especially subtle and early (pre-)malignant lesions in the esophagus and stomach can easily be missed by endoscopists (Fig. 1).

Meltem Tokat and Laurelle van Tilburg shared first authorship.
Correspondence to:

Manon C.W. Spaander, v.spaander@erasmusmc.nl 
Fig. 1. Endoscopic images of subtle early esophageal and gastric (pre-)malignant lesions of which detection rates can be increased with assistance of AI. The (pre-) malignant lesions are marked with a red rectangle. a Early BE neoplasia with WLE. b The same lesion as a with ME-NBI. c, d ESCC with WLE and ME-NBI. e, f EGC with WLE and ME-NBI. g, h GIM located at the angulus in the stomach with WLE and NBI. AI, artificial intelligence; BE, Barrett's esophagus; EGC, early gastric cancer; ESCC, esophageal squamous cell carcinoma; GI, gastrointestinal; GIM, gastric intestinal metaplasia; ME, magnified endoscopy; ME-NBI, magnified endoscopy and narrow band imaging; NBI, narrow band imaging; WLE, white light endoscopy.
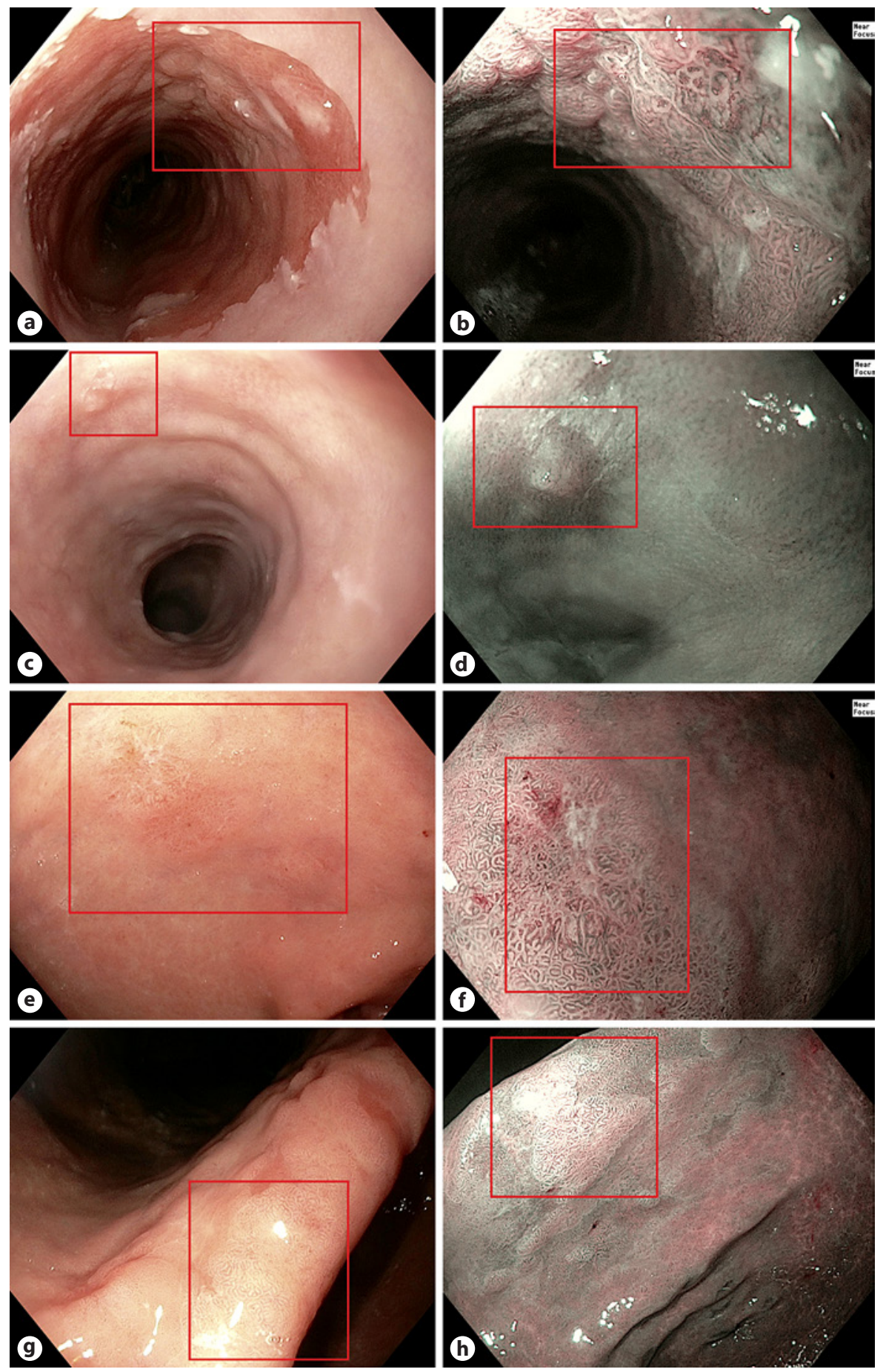

Artificial intelligence (AI) technology has the potential to overcome these obstacles. AI models have been introduced as a tool to aid in endoscopic detection, characterization, and delineation of premalignant and malignant lesions of the upper gastrointestinal (GI) tract [7-11]. Over the past decade, several AI systems have been devel- oped to assist endoscopists in the detection and staging of lesions in the upper GI tract. In this review, we aimed to provide an overview of the possible indications of AI systems in upper GI endoscopy (shown in Fig. 2) and hypothesize about potential challenges for its use in clinical practice. 
Fig. 2. Application of AI in upper GI endoscopy - topics that are addressed in this review. AI, artificial intelligence; $\mathrm{BE}$, Barrett's esophagus; CAG, chronic atrophic gastritis; EGC, early gastric cancer; ESCC, esophageal squamous cell carcinoma; GI, gastrointestinal; GIM, gastric intestinal metaplasia; HP, Helicobacter pylori.

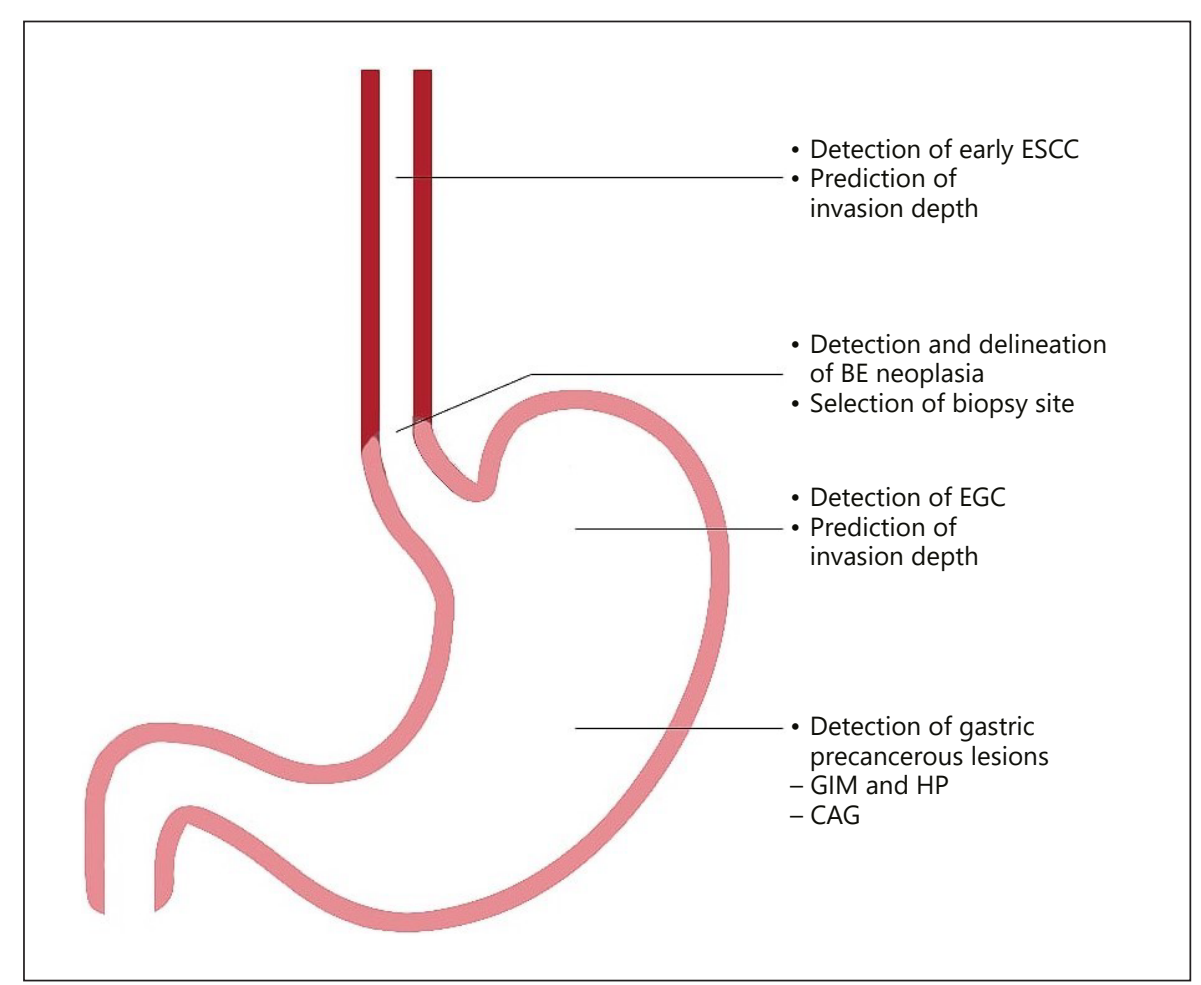

\section{Principles of Al}

AI refers to a machine-based intelligence which mimics human cognitive functions, such as learning and decision-making. Machine learning (ML) is a form of AI consisting of a teaching algorithm to recognize data patterns and utilize data to predict new data. In order to predict outcomes, an ML algorithm needs to be exposed to different example datasets. Deep learning (DL) is an advanced ML method, which uses layers of artificial neural networks to hierarchically structure data and extract features without human aid. Similar to the human brain, DL methods approach tasks by analyzing information from different concepts before assigning them to a specific class. Different from conventional ML algorithms that need human intervention to correct errors, DL has the ability to learn from its mistakes. This self-learning ability of DL technology makes it possible to increase its performance as exposure to data increases.

The most widely known DL method in endoscopy is based on convolutional neural network (CNN) and consists of a neural network architecture which is mainly used for image recognition and classification. To achieve sufficient diagnostic accuracy, a DL system needs to be trained and validated with large amounts of labeled data during different steps (shown in Fig. 3). First, the algorithm is subjected to a large dataset of mostly nonendoscopic labeled images. These labeled images are often obtained from open access databases, such as ImageNet [12]. Second, the algorithm needs to be trained and validated with a dataset of labeled endoscopic images. Last, when performance is sufficient, the algorithm needs to be tested. Computer-aided detection (CAD) systems in GI endoscopy are ML methods specifically developed to assist endoscopists to improve accurate detection and staging of pathology, including early stages of disease and selection of optimal biopsy sites.

\section{Esophagus}

\section{Neoplasia in Barrett's Esophagus}

The incidence of esophageal adenocarcinoma (EAC) is rapidly increasing in Western society $[13,14]$. Barrett's esophagus (BE) is a precancerous condition, which may progress to EAC [15]. Therefore, guidelines recommend endoscopic surveillance of BE in order to diagnose neoplastic progression in early stages. Endoscopic assessment of the esophagus with high-definition (HD) white light endoscopy (WLE) is advised to optimize the detec- 


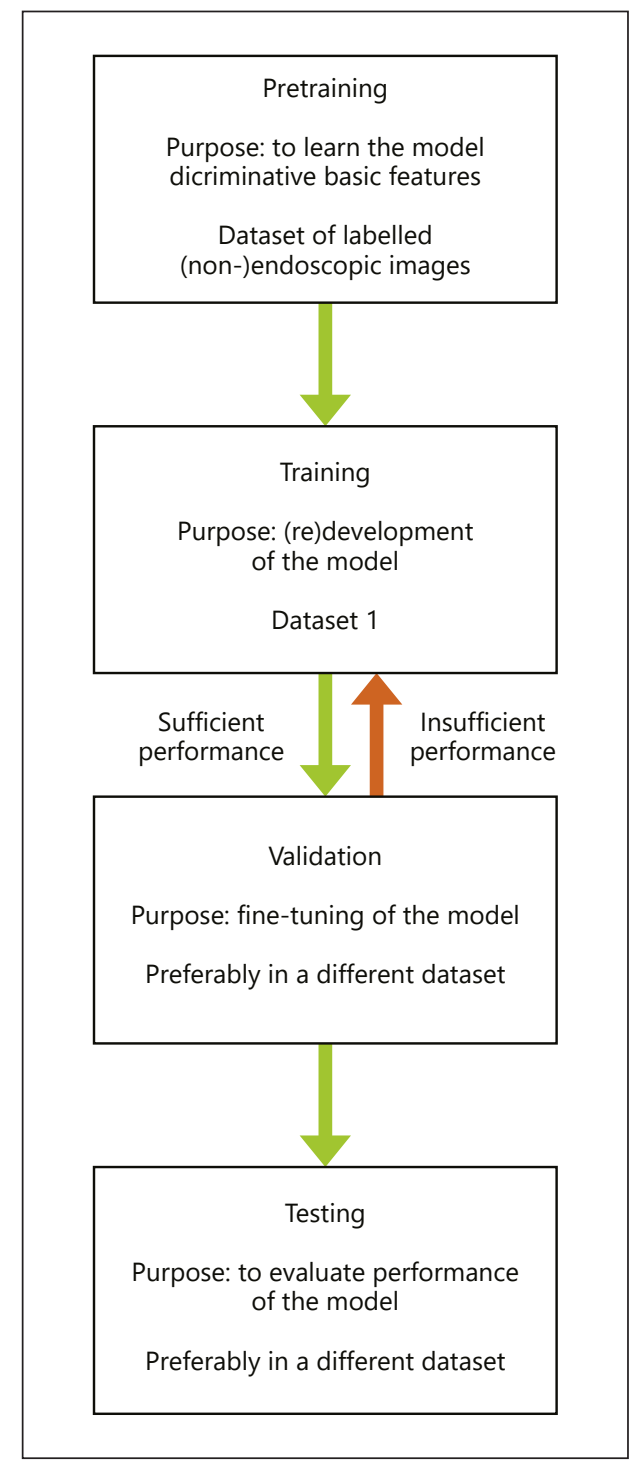

Fig. 3. Visual steps in the development of an AI model: pretraining, training, validation, and testing. AI, artificial intelligence.

tion of dysplastic Barrett mucosa [1, 2]. Chromoendoscopy can be utilized to aid in detection of lesions; however, additional value to WLE has not been proven [16]. Given the low progression rate among BE patients, which is estimated at $0.5 \%$ per year, the majority of gastroenterologists never encounter dysplasia and therefore may be less familiar with the mucosal changes associated with presence of neoplasia [17]. Visible neoplastic lesions, including early EAC, may remain undetected, especially when endoscopic surveillance is performed by endoscopists with limited experience in the recognition of early neoplastic lesions [18, 19]. Low-grade dysplasia may present itself with very subtle mucosal changes and is therefore easily missed [6]. To increase the diagnostic yield of dysplasia, guidelines recommend taking 4-quadrant biopsies at each 2 -cm interval of the Barrett segment, known as the Seattle protocol [20]. Combined with WLE, it is estimated that up to $90 \%$ of high-grade dysplasia (HGD) and EAC cases are detected [21]. Nevertheless, adherence to this protocol is poor as it is a time-consuming procedure, especially in patients with a long-segment BE [22].

AI in the Detection of Barrett Neoplasia

Several ML methods were developed to aid in diagnosis of BE neoplasia (Table 1). The majority of studies evaluated diagnostic performance of $\mathrm{CNN}$ algorithms in WLE images [7, 10, 23-27]. Hashimoto et al. [25] developed an algorithm based on CNN technology to aid in the detection of Barrett neoplasia by image annotation of areas suspect for neoplasia. The pretrained algorithm was trained with 916 images of BE patients with HGD and early EAC. The CNN then analyzed 225 images of dysplastic BE and 233 of nondysplastic Barrett's esophagus (NDBE) images with $95 \%$ accuracy. The ARGOS consortium performed several studies with $\mathrm{AI}$ algorithms to aid in the detection, characterization, and delineation of $\mathrm{BE}$ neoplasia and to improve the selection of biopsy sites [7, 23, 27, 28]. De Groof et al. [7] developed an AI model based on prospectively collected WLE images for the detection and delineation of BE neoplasia with a sensitivity, specificity, and accuracy of $95 \%, 85 \%$, and $92 \%$, respectively. Application of CAD in detection of Barrett neoplasia is also being explored in NBI images and videos [25, 26,28 ]. Struyvenberg et al. [28] developed a CAD system using 30,021 NBI video frames (average video consisted of 250 fragments obtained during $10 \mathrm{~s}$ of video) and detected BE neoplasia with accuracy of $83 \%$.

Recently, the first prospective studies during live endoscopic procedures were performed by de Groof et al. [24] and Ebigbo et al. [10]. De Groof et al. [23] trained their CAD model with 1,704 high-resolution images of 669 patients with histologically confirmed Barrett neoplasia or NDBE. Algorithm performance was externally validated with separate datasets, each containing $80 \mathrm{im}$ ages which were also scored for the presence of dysplasia by 53 general endoscopists. The CAD system classified images as dysplastic or nondysplastic with $90 \%$ sensitivity, $88 \%$ specificity, and $89 \%$ accuracy. The AI model outperformed the endoscopists in detection of early Barrett neoplasia in another dataset containing 80 images as the sensitivity, specificity, and accuracy of the CAD system 


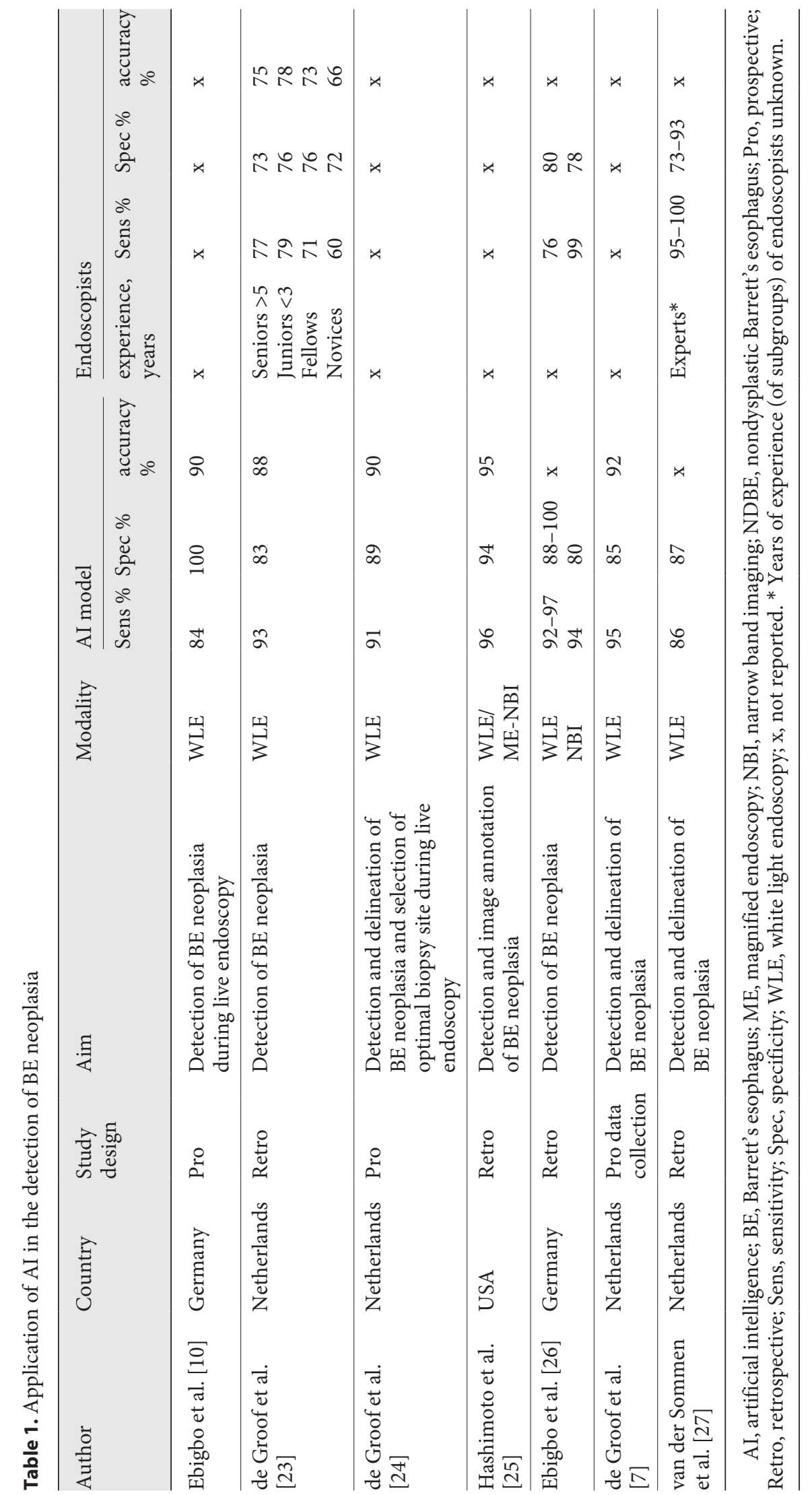


and endoscopists was, respectively, $93 \%$ versus $72 \%, 83 \%$ versus $74 \%$, and $88 \%$ versus $73 \%$ [23]. The CAD model was tested during real-time endoscopy with an accuracy of $90 \%$ [24]. Ebigbo et al. [26] developed a CAD-DL system based on 148 HD-WLE and NBI images of 33 early EAC and 41 NDBE areas in one database and $100 \mathrm{HD}$ WLE images of 17 early EAC and $22 \mathrm{NDBE}$ areas in a second database. Based on the images in these two datasets, the AI model reached a $92-97 \%$ sensitivity and 88 $100 \%$ specificity for WLE images and $94 \%$ sensitivity and $80 \%$ specificity for NBI images. Afterwards, the developed CNN-CAD algorithm was tested during real-time daily endoscopy in 14 patients with BE neoplasia with an accuracy of $89.9 \%$ [10]. The majority of previous mentioned studies showed high accuracy of AI models in the detection of BE neoplasia. Main limitations of these studies were the retrospective design and small sample size.

\section{Esophageal Squamous Cell Carcinoma}

Squamous cell carcinoma remains the predominant histologic type of esophageal cancer (EC), which accounts for $80 \%$ of the cases worldwide $[29,30]$. The incidence rates of esophageal squamous cell carcinoma (ESCC) vary strongly among geographic regions, with highest rates in Eastern Asia [29]. Most ESCC are detected in advanced stages and therefore associated with a poor 5-year survival rate of merely $20 \%$ [31]. The prognosis of early ESCC is considerably better, since the risk of lymph node and distant metastasis is associated with the tumor invasion depth [32]. Additional lugol's iodine staining or WLE and NBI can be used to increase the detection of subtle esophageal lesions $[33,34]$. The combination of magnification and NBI during endoscopy (ME-NBI) allows visualization of the microvasculature of the esophageal epithelium, which can be classified according to the intrapapillary capillary loop (IPCL) classification [35]. This classification can help differentiate dysplasia from nondysplasia in daily clinical practice [36].

\section{AI in the Detection of ESCC}

Most studies that investigated AI for the early detection of ESCC derive from Asian countries [29, 37-43]. AI models based on CNN during WLE are mostly investigated to detect squamous dysplasia and early ESCC (shown in Table 2) [37-41]. Horie et al. [9] developed a CNN-CAD system for the detection of EC (both ESCC and EAC; 8,428 images for system development and 1,118 images for validation). This study showed that $\mathrm{CNN}$ CAD can correctly detect EC cases, including both superficial and advanced cancers with a sensitivity of $98 \%$. Fur- thermore, the CNN-CAD system was accurately able to detect small cancerous lesions $<10 \mathrm{~mm}$ that can be easily missed, even by experienced endoscopists. Shimamoto et al. [41] compared the use of DL during WLE and during NBI for the accurate detection of the invasion depth in ESCC. The accuracy was higher in WLE than that in MENBI (98.7\% vs. 89.2\%) [41]. Ohmori et al. [37] showed that their AI system had a high sensitivity for the detection of ESCC using non-ME NBI and high accuracy for the differentiation of ESCC from noncancerous lesions.

Endoscopic screening and detection of ESCC remains challenging partly because it is liable to the interobserver variability between endoscopists [35]. Early-stage ESCC are difficult to detect, especially for trainee endoscopists (sensitivity of NBI for ESCC detection in trainee vs. expert endoscopists: $53 \%$ vs. $100 \%$ ) [45]. Several studies compared diagnostic parameters of developed AI models to endoscopists [37-42, 44]. Cai et al. [38] developed a CNN-CAD system based on WLE (2,428 images from 746 patients for training and 187 images from 52 patients for validation) which was compared to 3 groups of endoscopists (seniors with $>15$ years of experience, mid-levels with $5-15$ years of experience, and juniors with $<5$ years of experience). Sensitivity of AI for detection of ESCC appeared to be higher, even for the experienced endoscopists. The sensitivity of the AI system versus senior, midlevel, and junior endoscopists was $97.8 \%$ versus $86.3 \%$, $78.6 \%$, and $61.9 \%$, respectively. Zhao et al. [42] developed a CAD model based on ME-NBI to investigate the automated classification of IPCLs. The mean diagnostic accuracy of the CAD system was higher than that of midlevel and junior endoscopists for the detection of malignant esophageal lesions $(p<0.001)$. Fukuda and colleagues [44] divided the diagnostic process into 2 parts: detection (identify suspicious lesions) and characterization (differentiate cancer from no cancer). The developed CNN-DL system had a better diagnostic performance than the expert endoscopists [44]. Major limitations of these studies included the small sample size of images used for both training [38, 42] and validation [37, 38, 42, 44]. Furthermore, the samples of participating endoscopists with different levels of endoscopic experience were relatively small, ranging from 4 to 15 endoscopists per subgroup.

\section{AI in Prediction of Invasion Depth of ESCC}

The tumor invasion depth is an important prognostic factor in ESCC [46]. Accurate endoscopic detection of the invasion depth is essential for decision-making between endoscopic resection or proceeding to esophagectomy with lymphadenectomy [47]. To optimize endoscopic 


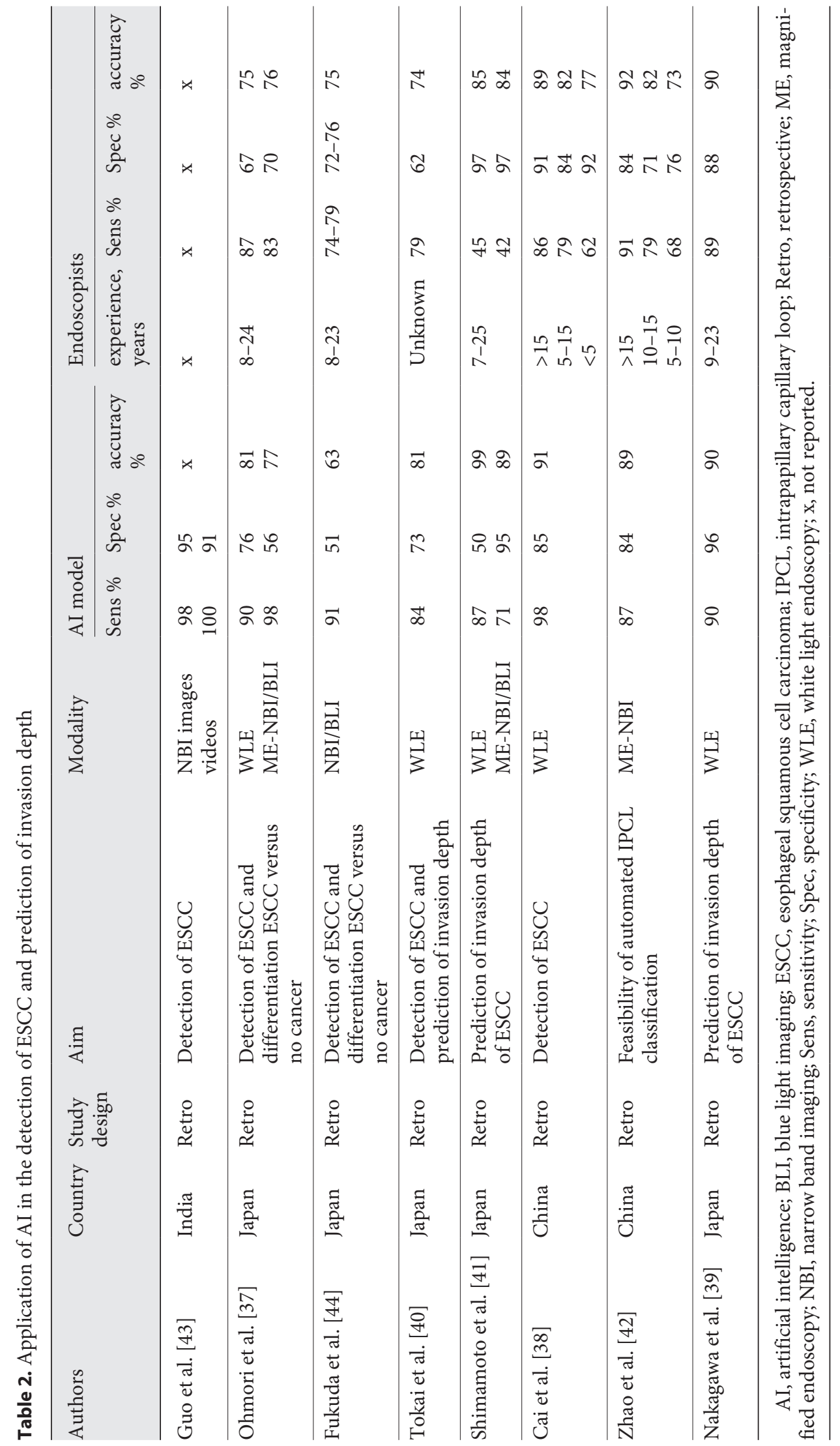


prediction of invasion depth, the role of AI was studied [39-41]. Shimamoto et al. [41] developed an AI system on WLE and NBI images from endoscopic videos to estimate the invasion depth, which was compared to experienced endoscopists (7-25 years of experience). The AI model outperformed the endoscopists in both non-ME and ME-NBI with a sensitivity, specificity, and accuracy of AI versus endoscopists using ME-NBI of 71\%, 95\%, and $89 \%$ versus $42 \%, 97 \%$, and $84 \%$, respectively. Tokai and colleagues [40] developed an AI model to predict the ESCC invasion depth on 1,751 images, which was validated on 291 images. The diagnostic accuracy of the AI model outperformed 12 out of 13 endoscopists [40].

\section{Stomach}

Gastric Precancerous Lesions and Early Gastric Cancer

Helicobacter pylori (HP) infection can cause chronic atrophic gastritis (CAG) and gastric intestinal metaplasia (GIM), which are both precancerous conditions associated with an increased risk of gastric cancer (GC) development $[3,48]$. GC is often diagnosed in an advanced stage, with an estimated 5-year survival rate of 20\% [30]. Endoscopic surveillance is offered to patients with CAG and GIM to detect GC in an early stage as detection of early GC (EGC) improves survival [3]. Current surveillance strategies consist of adequate inspection of the gastric mucosa and standardized random biopsy sampling according to the Sydney protocol for topographic mapping [3]. Guidelines recommend use of HD-chromoendoscopy in GC surveillance as it improves optical diagnosis of precancerous lesions and EGC [3,49-51]. The treatment strategy is determined by the invasion depth, which is an important prognostic factor in EGC $[3,30]$. In early cases, diagnosis of EGC can be difficult as features can be subtle and EGC is easily missed in the presence of other pathology such as gastritis. AI models may improve the diagnostic accuracy by locating areas suspect for cancer and aid the endoscopist in detection and staging of gastric pathology.

\section{AI in the Detection of EGC}

The application of AI for the detection of EGC has been investigated in WLE images [52-57] and optic chromoendoscopy images (Table 3) [8,58-63]. Li et al. [8] developed a CNN model on 386 images of benign lesions and 1,702 images of EGC for model development and 171 images of noncancerous lesions, and 170 EGC images to test the models' performance. The AI model had a diag- nostic accuracy of $91 \%$ versus $87 \%$ when used by experts and $70-74 \%$ for nonexpert endoscopists. Horiuchi et al. [59] tested a CAD system to detect EGC using 174 NBI videos that contained 87 cancerous lesions. The CAD system was trained with 2,570 images containing cancerous and noncancerous gastric lesions. The performance of the CAD system was benchmarked against 11 endoscopists with experience in NBI and showed varying results. Only 2 endoscopists were outperformed by the CAD system. Similar results were found in the study of Ikenoyama et al. [55] that assessed the application of AI in detecting GC with both WLE and NBI.

AI in Prediction of Invasion Depth of EGC

Few research groups have developed CAD systems to assess the invasion depth of EGC $[52,56,60]$. Nagao et al. [60] developed a CNN-CAD system by using 16,557 images of 1,084 GC cases that underwent endoscopic resection or radical surgery, to study if invasion depth of EGC can be determined. Prediction of invasion depth was analyzed in both WLE and NBI modality. The CAD system predicted the invasion depth with a sensitivity of $84 \%$ and $75 \%$, specificity of $99 \%$ and $100 \%$, and accuracy of $94 \%$ and $94 \%$ during WLE and NBI images, respectively. Yoon et al. [52] analyzed 11,539 images of both GC (T1a and T1b) and non-EGC and predicted the invasion depth with an AUC of 0.85 . However, in case of undifferentiated histology, the accuracy of the AI model was significantly lower. Despite the high performance of the CAD systems, only images were used to train and calculate performance of the algorithm, and video analysis has yet to be tested.

AI in Detection of Gastric Precancerous Lesions and HP Infection

Recent AI systems developed to enhance endoscopic detection of gastric precancerous lesions and HP are shown in Table 4 [11, 64-71]. In 2 studies, AI models were compared to endoscopists with different levels of experience in detection of CAG [11, 64]. Zhang et al. [64] designed a CNN model to detect CAG by using 5,470 antrum images of 1,699 patients. Images were classified as mild, moderate, and severe CAG. CAG was histologically confirmed in 3,042 images. The performance of the CNN model was compared to 3 expert endoscopists. The model outperformed the endoscopists with a sensitivity, specificity, and accuracy of $95 \%, 94 \%$, and $94 \%$, respectively. Highest detection rate was seen in severe CAG, with an accuracy of 99\%. Guimarães et al. [11] showed similar results and reported a 93\% accuracy for the detection of CAG in WLE images of the proximal stomach. Yan and colleagues [65] 


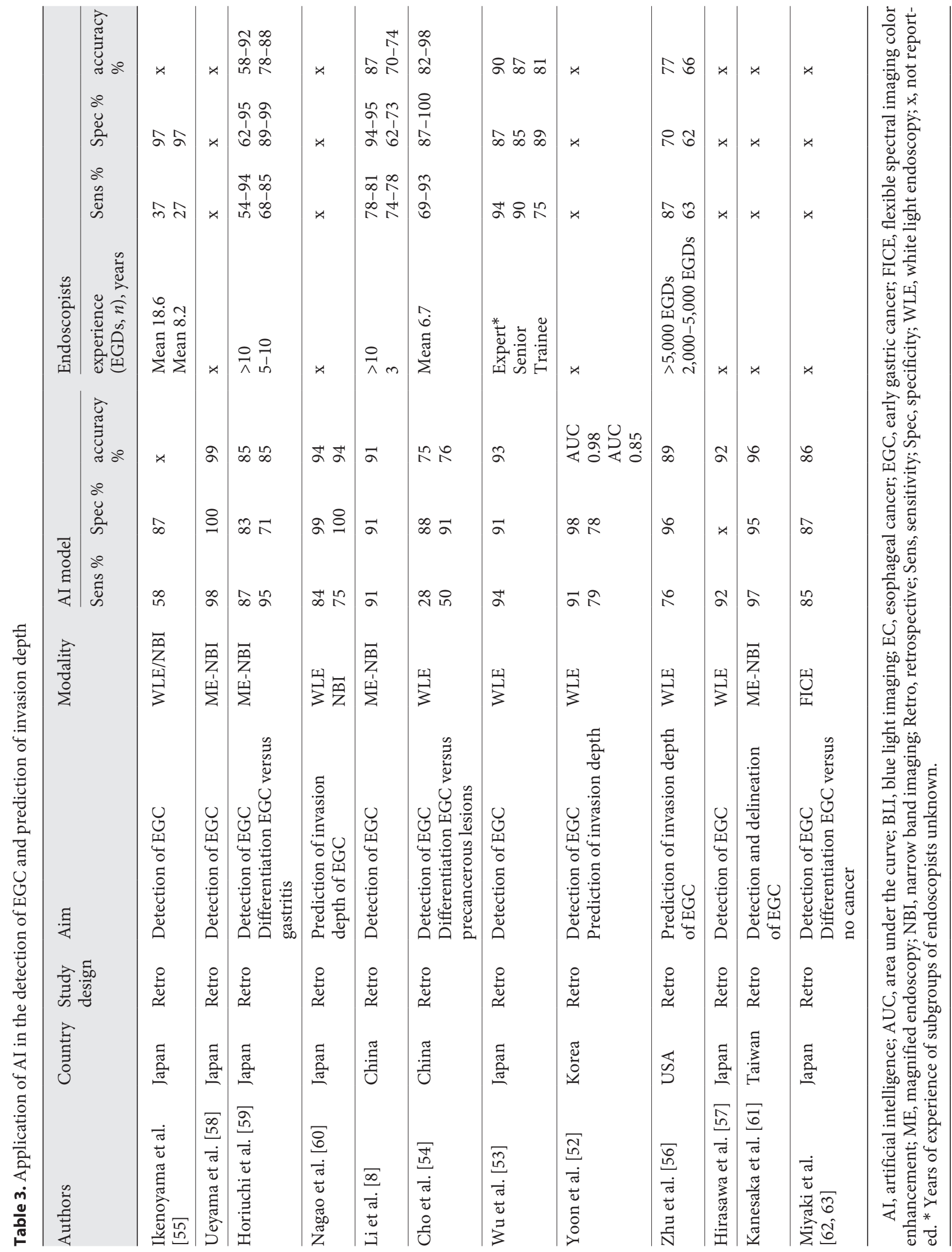




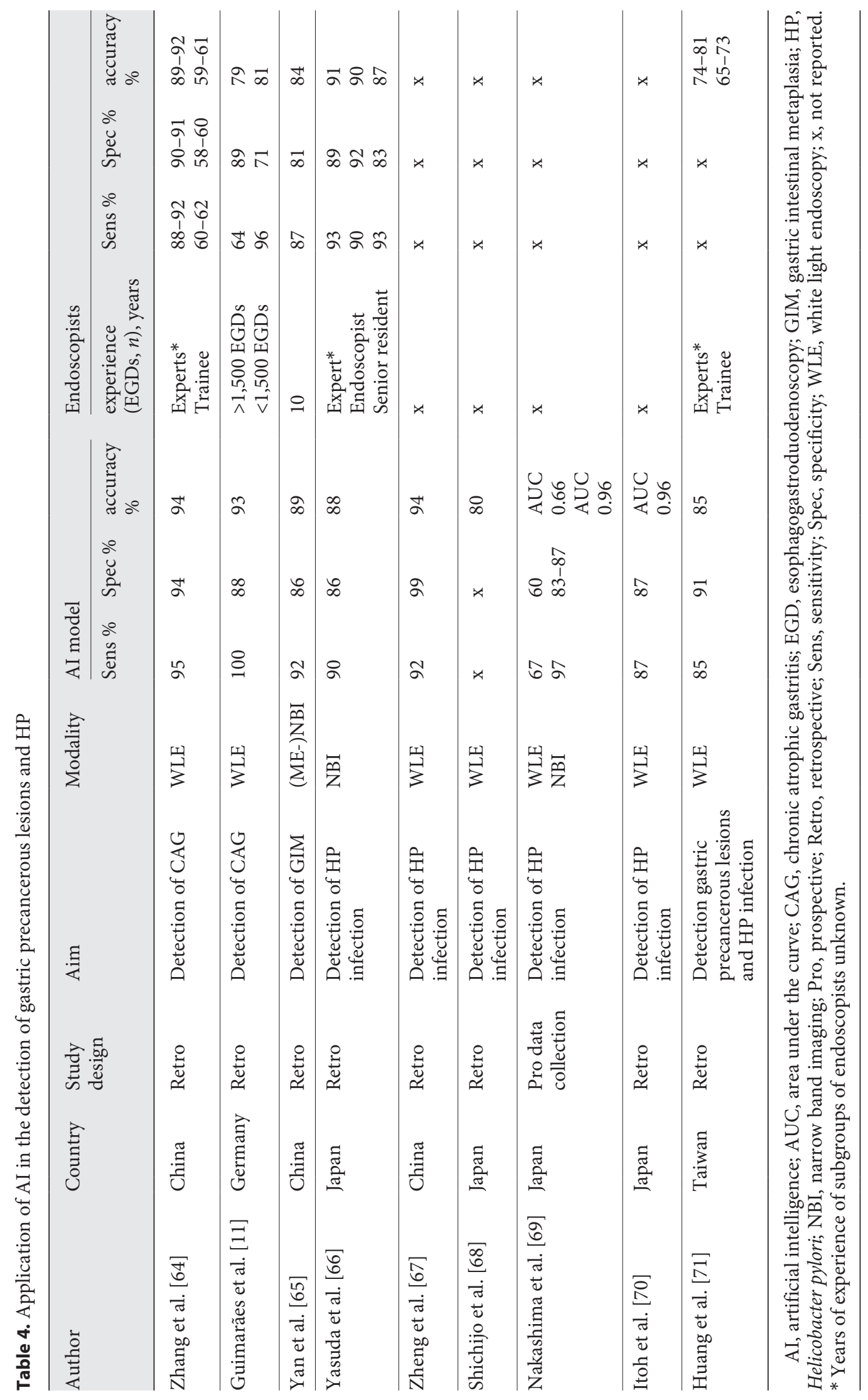


Table 5. Current status of (the development of) AI systems per upper GI indication

\begin{tabular}{|c|c|}
\hline $\begin{array}{l}\text { Indications for AI in upper GI } \\
\text { endoscopy }\end{array}$ & Current status of AI systems \\
\hline BE neoplasia & Algorithms are trained and validated with a dataset of labeled endoscopic images \\
\hline Selection of biopsy site & $\begin{array}{l}\text { Next step: Validation of AI algorithms in large groups of patients during live endoscopic } \\
\text { procedures. Assess AI performance when used by endoscopists with different levels of experience }\end{array}$ \\
\hline Detection of early ESCC & Retrospective studies with high quality images or videos haven been performed \\
\hline Prediction of invasion depth & Next step: Prospective data collection of images and videos \\
\hline EGC & Algorithms are trained and validated with a dataset of labeled endoscopic images \\
\hline Detection of EGC & Retrospective studies with high quality images or videos \\
\hline Prediction of invasion depth & Next step: Prospective data collection of images and videos \\
\hline
\end{tabular}

AI, artificial intelligence; BE, Barrett's esophagus; CAG, chronic atrophic gastritis; EGC, early gastric cancer; ESCC, esophageal squamous cell carcinoma; GI, gastrointestinal; GIM, gastric intestinal metaplasia; HP, Helicobacter pylori.

developed a CNN-CAD model for the detection of GIM with ME-NBI. The AI model reported a diagnostic accuracy of $89 \%$ with an accuracy of $84 \%$ for expert endoscopists with 10 years of endoscopic experience $(p=0.42)$.

Zheng et al. [67] developed a CAD system to determine HP infection status, based on endoscopic images. In total, 15,484 gastric images of 1,959 patients of which 1,157 with a HP infection were used. This study aimed to investigate whether the AI model could accurately diagnose HP infection during endoscopy without the need for biopsies. The CNN system showed a high performance with an accuracy of $92 \%$. Nakashima et al. [69] used a DL model to diagnose HP infection with the use of WLE and blue light imaging (BLI). The research group conducted a single-center prospective study with 222 participants of which 105 had a confirmed HP infection. The DL model had an AUC of 0.96 with BLI. However, with WLE images, the AUC of the AI model decreased to 0.66 .

\section{Conclusion and Potential Challenges of Implementing AI Upper Endoscopy into Clinical Practice}

In this review, we have shown that AI systems have been applied in upper GI endoscopy for several indications: (1) detection, characterization, and delineation of esophageal and GC and their premalignant conditions; (2) prediction of tumor invasion; and (3) diagnosis of a HP infection. The current status of AI models for each indication in upper GI endoscopy is shown in Table 5. So far, all AI studies in upper GI endoscopy have shown promising results with high performance for accurate detection and staging of (pre-)malignant lesions in both the esophagus and stomach. The benefit, especially on the quality of endoscopy by the use of AI in upper GI however, still needs to be demonstrated and may differ between endoscopists based on their skills and experience.

The use of AI in upper GI endoscopy may be of additional value for clinical practice for different reasons. AI has the potential to provide real-time assistance by red flagging cancers that remained undetected by endoscopists and may improve the yield of biopsies by indicating the optimal biopsy sites during live endoscopic procedures. More accurate prediction of tumor invasion of early-stage cancers may improve the selection of patients eligible for endoscopic resection and may prevent unnecessary invasive surgery. And more accurate endoscopic diagnosis of HP infection and gastric precancerous lesions by AI models may replace gastric biopsies.

To date, most AI models in upper GI endoscopy are developed in an ideal setting with high-quality imagery. This setting does not always reflect real-life endoscopy, 
where good visualization of the mucosa depends on the experience and skills of the endoscopists, which is essential for optimal performance of AI. Although several studies compared AI models to endoscopists, studies reporting on the diagnostic performance of AI models for each experience level of endoscopists are scarce. The outcome of these studies will better illuminate for which indication AI may be of additional value in relation to endoscopist's own experience and skills. For example, in GC, AI outperformed mid-level and trainee but not expert endoscopists. Besides studies linking the performance of AI models to endoscopists with different levels of experience, studies that investigate AI during real-time upper GI endoscopy are still very scarce. To date, no AI systems have been validated in large groups of patients during live endoscopic procedures. Large prospective trials are awaited for to validate the additional value and confirm the clinical significance of AI models during reallife endoscopy.

In conclusion, AI models in upper GI endoscopy showed high diagnostic performance for the detection, characterization, and delineation of upper GI lesions. In addition, AI shows promising results in the prediction of the tumor invasion depth and diagnosis of HP. The benefit of AI correlated to endoscopist skills and experience need to be further addressed, while prospective studies are needed to confirm its accuracy and feasibility during real-time daily endoscopy.

\section{Conflict of Interest Statement}

M.T. has no conflict of interest to declare. L.T. received research support from Dr Falk Pharma. A.D.K. received research support from Dr. Falk Pharma and consultancy fees from ERBE Elektromedizin and Pentax Medical. M.C.W.S. received research support from Medtronics, Boston Scientific, Norgine, Informed, Sentinel, and Sysmex.

\section{Funding Sources}

No funding.

\section{Author Contributions}

M.C.V.S. was invited for this review and contributed to, with M.T., the conception and design; M.T. and L.T. jointly acted as first authors of this work, screened articles for inclusion in this review, interpreted data, and drafted the manuscript. A.D.K. co-authored the manuscript. All the authors critically edited, read, and approved the final manuscript.

\section{References}

1 Weusten B, Bisschops R, Coron E, Dinis-Ribeiro M, Dumonceau J-M, Esteban J-M, et al. Endoscopic management of Barrett's esophagus: European Society of Gastrointestinal Endoscopy (esge) position statement. Endoscopy. $2017 \mathrm{Feb} ; 49(2): 191-8$.

2 Qumseya B, Sultan S, Bain P, Jamil L, Jacobson B, Anandasabapathy S, et al. ASGE guideline on screening and surveillance of Barrett's esophagus. Gastrointest Endosc. 2019;90: 335-59.e2.

3 Pimentel-Nunes P, Libânio D, Marcos-Pinto R, Areia M, Leja M, Esposito G, et al. Management of epithelial precancerous conditions and lesions in the stomach (maps ii): European Society of Gastrointestinal Endoscopy (esge), European helicobacter and microbiota study group (ehmsg), European Society of $\mathrm{Pa}$ thology (esp), and sociedade Portuguesa de endoscopia digestiva (sped) guideline update 2019. Endoscopy. 2019;51:365-88.

4 Lordick F, Mariette C, Haustermans K, Obermannová R, Arnold D, Committee EG. Oesophageal cancer: ESMO clinical practice guidelines for diagnosis, treatment and follow-up. Ann Oncol. 2016;27:v50-7.
5 Kerkhof M, Van Dekken H, Steyerberg EW, Meijer GA, Mulder AH, de Bruïne A, et al. Grading of dysplasia in Barrett's oesophagus: substantial interobserver variation between general and gastrointestinal pathologists. Histopathology. 2007;50:920-7.

6 Curvers WL, ten Kate FJ, Krishnadath KK, Visser M, Elzer B, Baak LC, et al. Low-grade dysplasia in Barrett's esophagus: overdiagnosed and underestimated. Am J Gastroenterol. 2010;105:1523-30.

7 de Groof J, van der Sommen F, van der Putten J, Struyvenberg MR, Zinger S, Curvers WL, et al. The argos project: the development of a computer-aided detection system to improve detection of Barrett's neoplasia on white light endoscopy. United European Gastroenterol J. 2019;7:538-47.

8 Li L, Chen Y, Shen Z, Zhang X, Sang J, Ding $\mathrm{Y}$, et al. Convolutional neural network for the diagnosis of early gastric cancer based on magnifying narrow band imaging. Gastric Cancer. 2020;23:126-32.

9 Horie Y, Yoshio T, Aoyama K, Yoshimizu S, Horiuchi Y, Ishiyama A, et al. Diagnostic outcomes of esophageal cancer by artificial intelligence using convolutional neural networks. Gastrointest Endosc. 2019;89:25-32.
10 Ebigbo A, Mendel R, Probst A, Manzeneder J, Prinz F, de Souza LA Jr, et al. Real-time use of artificial intelligence in the evaluation of cancer in Barrett's oesophagus. Gut. 2020;69:615-6.

11 Guimaraes P, Keller A, Fehlmann T, Lammert F, Casper M. Deep-learning based detection of gastric precancerous conditions. Gut. 2020; 69:4-6.

12 Deng J, Dong W, Socher R, Li L-J, Li K, Fei-Fei L . Imagenet: a large-scale hierarchical image database. In: 2009 IEEE conference on computer vision and pattern recognition. 2009. p. 248-55.confpap.

13 Steevens J, Botterweck AA, Dirx MJ, van den Brandt PA, Schouten LJ. Trends in incidence of oesophageal and stomach cancer subtypes in europe. Eur J Gastroenterol Hepatol. 2010; 22:669-78.

14 Cook MB, Chow WH, Devesa SS. Oesophageal cancer incidence in the United States by race, sex, and histologic type, 1977-2005. Br J Cancer. 2009;101:855-9.

15 Duits LC, van der Wel MJ, Cotton CC, Phoa KN, Ten Kate FJW, Seldenrijk CA, et al. Patients with Barrett's esophagus and confirmed persistent low-grade dysplasia are at increased risk for progression to neoplasia. Gastroenterology. 2017;152:993-1001.e1. 
16 Ngamruengphong S, Sharma VK, Das A. Diagnostic yield of methylene blue chromoendoscopy for detecting specialized intestinal metaplasia and dysplasia in Barrett's esophagus: a meta-analysis. Gastrointest Endosc. 2009;69:1021-8.

17 Yousef F, Cardwell C, Cantwell MM, Galway $\mathrm{K}$, Johnston BT, Murray L. The incidence of esophageal cancer and high-grade dysplasia in Barrett's esophagus: a systematic review and meta-analysis. Am J Epidemiol. 2008;168: 237-49.

18 Noordzij IC, Van Loon van de Ende MCM, Curvers WL, van Lijnschoten G, Huysentruyt CJ, Schoon EJ. Dysplasia in random biopsies from Barrett's surveillance is an important marker for more severe pathology. Dig Dis Sci. 2021;66:1-8.

19 Schölvinck DW, Van Der Meulen K, Bergman JJGHM, Weusten BLAM. Detection of lesions in dysplastic Barrett's esophagus by community and expert endoscopists. Endoscopy. 2017;49:113-20.

20 American Gastroenterological Association; Spechler SJ, Sharma P, Souza RF, Inadomi JM, Shaheen NJ. American gastroenterological association technical review on the management of Barrett's esophagus. Gastroenterology. 2011;140:e18-52. quiz e13.

21 Konda VJ, Ross AS, Ferguson MK, Hart JA, Lin S, Naylor K, et al. Is the risk of concomitant invasive esophageal cancer in high-grade dysplasia in Barrett's esophagus overestimated? Clin Gastroenterol Hepatol. 2008;6:15964.

22 Roumans CAM, van der Bogt RD, Steyerberg EW, Rizopoulos D, Lansdorp-Vogelaar I, Sharma P, et al. Adherence to recommendations of Barrett's esophagus surveillance guidelines: a systematic review and metaanalysis. Endoscopy. 2020;52:17-28.

23 de Groof AJ, Struyvenberg MR, van der Putten J, van der Sommen F, Fockens KN, Curvers WL, et al. Deep-learning system detects neoplasia in patients with Barrett's esophagus with higher accuracy than endoscopists in a multistep training and validation study with benchmarking. Gastroenterology. 2020;158: 915-29.e4.

24 de Groof AJ, Struyvenberg MR, Fockens KN, van der Putten J, van der Sommen F, Boers TG, et al. Deep learning algorithm detection of Barrett's neoplasia with high accuracy during live endoscopic procedures: a pilot study (with video). Gastrointest Endosc. 2020;91: 1242-50.

25 Hashimoto R, Requa J, Dao T, Ninh A, Tran E, Mai D, et al. Artificial intelligence using convolutional neural networks for real-time detection of early esophageal neoplasia in Barrett's esophagus (with video). Gastrointest Endosc. 2020;91:1264-71.e1.

26 Ebigbo A, Mendel R, Probst A, Manzeneder J, Souza LA Jr, Papa JP, et al. Computer-aided diagnosis using deep learning in the evaluation of early oesophageal adenocarcinoma. Gut. 2019;68:1143-5.
27 van der Sommen F, Zinger S, Curvers WL, Bisschops R, Pech O, Weusten BL, et al. Computer-aided detection of early neoplastic lesions in Barrett's esophagus. Endoscopy. 2016;48:617-24.

28 Struyvenberg MR, de Groof AJ, van der Putten J, van der Sommen F, Baldaque-Silva F, Omae $\mathrm{M}$, et al. A computer-assisted algorithm for narrow-band imaging-based tissue characterization in Barrett's esophagus. Gastrointest Endosc. 2020;93(1):89-98.

29 Arnold M, Ferlay J, van Berge Henegouwen MI, Soerjomataram I. Global burden of oesophageal and gastric cancer by histology and subsite in 2018. Gut. 2020;69:1564-71.

30 Bray F, Ferlay J, Soerjomataram I, Siegel RL, Torre LA, Jemal A. Global cancer statistics 2018: globocan estimates of incidence and mortality worldwide for 36 cancers in 185 countries. CA Cancer J Clin. 2018;68:394424.

31 van Putten M, de Vos-Geelen J, Nieuwenhuijzen GAP, Siersema PD, Lemmens VEPP, Rosman C, et al. Long-term survival improvement in oesophageal cancer in the Netherlands. Eur J Cancer. 2018;94:138-47.

32 Henry MA, Lerco MM, Naresse LE, Crema E, Rodrigues MA. Outcome of superficial squamous cell carcinoma of the esophagus: a clinicopathological study. Acta Cir Bras. 2013;28: 373-8.

33 Lu P, Gu J, Zhang N, Sun Y, Wang J. Risk factors for precancerous lesions of esophageal squamous cell carcinoma in high-risk areas of rural china: a population-based screening study. Medicine. 2020;99:e21426.

34 Gruner M, Denis A, Masliah C, Amil M, Metivier-Cesbron E, Luet D, et al. Narrow-band imaging versus lugol chromoendoscopy for esophageal squamous cell cancer screening in normal endoscopic practice: randomized controlled trial. Endoscopy. 2020;53(7):67482.

35 Inoue $\mathrm{H}$, Honda $\mathrm{T}$, Nagai $\mathrm{K}$, Kawano T, Yoshino K, Takeshita K, et al. Ultra-high magnification endoscopic observation of carcinoma in situ of the esophagus. Dig Endosc. 1997; 9(1):16-8.

36 Inoue $H$, Kaga $M$, Ikeda $H$, Sato $C$, Sato $H$, Minami H, et al. Magnification endoscopy in esophageal squamous cell carcinoma: a review of the intrapapillary capillary loop classification. Ann Gastroenterol. 2015;28:41-8.

37 Ohmori M, Ishihara R, Aoyama K, Nakagawa $\mathrm{K}$, Iwagami H, Matsuura N, et al. Endoscopic detection and differentiation of esophageal lesions using a deep neural network. Gastrointest Endosc. 2020;91:301-9.e1.

38 Cai SL, Li B, Tan WM, Niu XJ, Yu HH, Yao $L Q$, et al. Using a deep learning system in endoscopy for screening of early esophageal squamous cell carcinoma (with video). Gastrointest Endosc. 2019;90:745-53.e2.

39 Nakagawa K, Ishihara R, Aoyama K, Ohmori M, Nakahira H, Matsuura N, et al. Classification for invasion depth of esophageal squamous cell carcinoma using a deep neural net- work compared with experienced endoscopists. Gastrointest Endosc. 2019;90:407-14.

40 Tokai Y, Yoshio T, Aoyama K, Horie Y, Yoshimizu S, Horiuchi Y, et al. Application of artificial intelligence using convolutional neural networks in determining the invasion depth of esophageal squamous cell carcinoma. Esophagus. 2020;17:250-6.

41 Shimamoto Y, Ishihara R, Kato Y, Shoji A, Inoue T, Matsueda K, et al. Real-time assessment of video images for esophageal squamous cell carcinoma invasion depth using artificial intelligence. J Gastroenterol. 2020;55:1037-45.

42 Zhao YY, Xue DX, Wang YL, Zhang R, Sun B, Cai YP, et al. Computer-assisted diagnosis of early esophageal squamous cell carcinoma using narrow-band imaging magnifying endoscopy. Endoscopy. 2019;51:333-41.

43 Guo L, Xiao X, Wu C, Zeng X, Zhang Y, Du J, et al. Real-time automated diagnosis of precancerous lesions and early esophageal squamous cell carcinoma using a deep learning model (with videos). Gastrointest Endosc. 2020;91:41-51.

44 Fukuda $\mathrm{H}$, Ishihara R, Kato $\mathrm{Y}$, Matsunaga $\mathrm{T}$, Nishida T, Yamada T, et al. Comparison of performances of artificial intelligence versus expert endoscopists for real-time assisted diagnosis of esophageal squamous cell carcinoma (with video). Gastrointest Endosc. 2020; 92:848-55.

45 Ishihara R, Takeuchi Y, Chatani R, Kidu T, Inoue T, Hanaoka N, et al. Prospective evaluation of narrow-band imaging endoscopy for screening of esophageal squamous mucosal high-grade neoplasia in experienced and less experienced endoscopists. Dis Esophagus. 2010;23:480-6.

46 Goda K, Irisawa A. Japan esophageal society classification for predicting the invasion depth of superficial esophageal squamous cell carcinoma: should it be modified now? Dig Endosc. 2020;32:37-8.

47 Domper Arnal MJ, Ferrández Arenas Á, Lanas Arbeloa Á. Esophageal cancer: risk factors, screening and endoscopic treatment in Western and Eastern countries. World J Gastroenterol. 2015;21:7933-43.

48 Correa P, Piazuelo MB. The gastric precancerous cascade. J Dig Dis. 2012;13:2-9.

49 Pimentel-Nunes P, Libânio D, Lage J, Abrantes D, Coimbra M, Esposito G, et al. A multicenter prospective study of the real-time use of narrow-band imaging in the diagnosis of premalignant gastric conditions and lesions. Endoscopy. 2016;48:723-30.

50 Banks M, Graham D, Jansen M, Gotoda T, Coda S, Di Pietro M, et al. British society of gastroenterology guidelines on the diagnosis and management of patients at risk of gastric adenocarcinoma. Gut. 2019;68:1545-75.

51 Kikuste I, Marques-Pereira R, Monteiro-Soares M, Pimentel-Nunes P, Areia M, Leja M, et al. Systematic review of the diagnosis of gastric premalignant conditions and neoplasia with high-resolution endoscopic technologies. Scand J Gastroenterol. 2013;48:1108-17. 
52 Yoon HJ, Kim S, Kim JH, Keum JS, Oh SI, Jo $\mathrm{J}$, et al. A lesion-based convolutional neural network improves endoscopic detection and depth prediction of early gastric cancer. J Clin Med. 2019;8:1310.

$53 \mathrm{Wu}$ L, Zhou W, Wan X, Zhang J, Shen L, Hu $S$, et al. A deep neural network improves endoscopic detection of early gastric cancer without blind spots. Endoscopy. 2019;51: 522-31.

54 Cho BJ, Bang CS, Park SW, Yang YJ, Seo SI, $\mathrm{Lim} \mathrm{H}$, et al. Automated classification of gastric neoplasms in endoscopic images using a convolutional neural network. Endoscopy. 2019;51:1121-9.

55 Ikenoyama Y, Hirasawa T, Ishioka M, Namikawa K, Yoshimizu S, Horiuchi Y, et al. Detecting early gastric cancer: comparison between the diagnostic ability of convolutional neural networks and endoscopists. Dig Endosc. 2021;33(1): 141-50.

56 Zhu Y, Wang QC, Xu MD, Zhang Z, Cheng J, Zhong YS, et al. Application of convolutional neural network in the diagnosis of the invasion depth of gastric cancer based on conventional endoscopy. Gastrointest Endosc. 2019; 89:806-15.e1.

57 Hirasawa T, Aoyama K, Tanimoto T, Ishihara S, Shichijo S, Ozawa T, et al. Application of artificial intelligence using a convolutional neural network for detecting gastric cancer in endoscopic images. Gastric Cancer. 2018;21: 653-60.

58 Ueyama H, Kato Y, Akazawa Y, Yatagai N, Komori H, Takeda T, et al. Application of artificial intelligence using a convolutional neural network for diagnosis of early gastric cancer based on magnifying endoscopy with narrow-band imaging. J Gastroenterol Hepatol. 2021;36(2):482-9.
59 Horiuchi Y, Hirasawa T, Ishizuka N, Tokai Y, Namikawa K, Yoshimizu S, et al. Performance of a computer-aided diagnosis system in diagnosing early gastric cancer using magnifying endoscopy videos with narrow-band imaging (with videos). Gastrointest Endosc. 2020;92: 856-65.e1.

60 Nagao S, Tsuji Y, Sakaguchi Y, Takahashi Y, Minatsuki C, Niimi K, et al. Highly accurate artificial intelligence systems to predict the invasion depth of gastric cancer: efficacy of conventional white-light imaging, nonmagnifying narrow-band imaging, and indigo-carmine dye contrast imaging. Gastrointest Endosc. 2020;92:866-73.e1.

61 Kanesaka T, Lee TC, Uedo N, Lin KP, Chen HZ, Lee JY, et al. Computer-aided diagnosis for identifying and delineating early gastric cancers in magnifying narrow-band imaging. Gastrointest Endosc. 2018;87:1339-44.

62 Miyaki R, Yoshida S, Tanaka S, Kominami Y, Sanomura Y, Matsuo T, et al. Quantitative identification of mucosal gastric cancer under magnifying endoscopy with flexible spectral imaging color enhancement. J Gastroenterol Hepatol. 2013;28:841-7.

63 Miyaki R, Yoshida S, Tanaka S, Kominami Y, Sanomura Y, Matsuo T, et al. A computer system to be used with laser-based endoscopy for quantitative diagnosis of early gastric cancer. J Clin Gastroenterol. 2015;49:108-15.

64 Zhang Y, Li F, Yuan F, Zhang K, Huo L, Dong $Z$, et al. Diagnosing chronic atrophic gastritis by gastroscopy using artificial intelligence. Dig Liver Dis. 2020;52:566-72.
65 Yan T, Wong PK, Choi IC, Vong CM, Yu HH. Intelligent diagnosis of gastric intestinal metaplasia based on convolutional neural network and limited number of endoscopic images. Comput Biol Med. 2020;126:104026.

66 Yasuda T, Hiroyasu T, Hiwa S, Okada Y, Hayashi S, Nakahata Y, et al. Potential of automatic diagnosis system with linked color imaging for diagnosis of helicobacter pylori infection. Dig Endosc. 2020;32:373-81.

67 Zheng W, Zhang X, Kim JJ, Zhu X, Ye G, Ye $B$, et al. High accuracy of convolutional neural network for evaluation of helicobacter pylori infection based on endoscopic images: preliminary experience. Clin Transl Gastroenterol. 2019;10:e00109.

68 Shichijo S, Endo Y, Aoyama K, Takeuchi Y, Ozawa T, Takiyama H, et al. Application of convolutional neural networks for evaluating helicobacter pylori infection status on the basis of endoscopic images. Scand J Gastroenterol. 2019;54:158-63.

69 Nakashima H, Kawahira H, Kawachi H, Sakaki N. Artificial intelligence diagnosis of helicobacter pylori infection using blue laser imaging-bright and linked color imaging: a single-center prospective study. Ann Gastroenterol. 2018;31:462-8.

70 Itoh T, Kawahira H, Nakashima H, Yata N. Deep learning analyzes helicobacter pylori infection by upper gastrointestinal endoscopy images. Endosc Int Open. 2018;6:E139-44.

71 Huang CR, Sheu BS, Chung PC, Yang HB. Computerized diagnosis of helicobacter pylori infection and associated gastric inflammation from endoscopic images by refined feature selection using a neural network. Endoscopy. 2004;36:601-8. 